\title{
EVOLUTION OF INTERACTIVE NICHE BREADTH AND ITS CONSEQUENCES IN PALEOECOSYSTEM NETWORKS
}

\author{
BURNS, Thomas P., Environmental Sciences Division, Oak Ridge National Laboratory, \\ Oak Ridge, TN 37831-6036, USA.
}

An ecological community, the living part of an ecosystem, can be conceived as a network of interacting organisms. This network specifies the biotic environment influencing the evolution and population dynamics of those organisms. The number and relative strengths of the interactions between an organism and the others in its environment defined by that network (termed network fittedness) are determined in part by heritable morphological and behavioral traits of the organism. This property, fittedness, can be quantified using Levins' (1968) measure of niche breadth applied to the probabilities of interacting with the other species of organisms in the network, i.e., fittedness is a measure of interactive niche breadth. It is hypothesized that organisms optimize fittedness and that the optimum increases with the variability of the environment.

These two hypotheses are combined in a model that predicts the dynamics of interactive structure of ecosystems and the fittednesses of their component organisms, both of which arise from the evolution and coevolution of populations of organisms and their ecological dynamics, including immigration and local extinction. This model applies to interactions of all sorts: predation, competition, mutualisms, etc.

This model leads to predictions on the evolution and stasis of organisms' morphological and behavioural traits and on how the variance in density of individual taxa change through a coherent spatiotemporal interval experiencing relatively undisturbed abiotic environmental conditions. The model predicts that, after an initial period of high turnover of species and dominance by species with high fittedness (generalists), the community will come to comprise an increasing proportion of organisms with narrow interactive niche breadths (specialists), the turnover of generalists will increase as they are replaced by specialists or they evolve specializations reflected in their morphology or behavior, and the turnover of specialist species will decrease. Population densities will fluctuate less as surviving species become more and more likely to interact with fewer species which also fluctuate less. Assuming an inverse relationship between fittedness and the sensitivity of populations to fluctuations in density of the species with which they interact, decreasing fittedness of the species in the community will lead to increasing sensitivity to the inevitable and unpredictable biotic or abiotic environmental perturbations, such as species invasions or increasing sedimentation rates. Moreover, the set of species forming the community will be increasingly sensitive to relatively small perturbations the greater the interval between perturbations, such that the timing of perturbations becomes important in determining the severity of the community's response. These predictions should be testable in the fossil record. 